\title{
Self-consistent Skyrme quasiparticle random-phase approximation for use in axially symmetric nuclei of arbitrary mass
}

\author{
J. Terasaki and J. Engel \\ Department of Physics and Astronomy, University of North Carolina, Chapel Hill, North Carolina 27599, USA
}

(Received 31 May 2010; published 27 September 2010)

\begin{abstract}
We describe a new implementation of the quasiparticle random-phase approximation (QRPA) in axially symmetric deformed nuclei with Skyrme and volume-pairing energy-density functionals. After using a variety of tests to demonstrate the accuracy of the code in ${ }^{24,26} \mathrm{Mg}$ and ${ }^{16} \mathrm{O}$, we report the first fully self-consistent application of the Skyrme QRPA to a heavy deformed nucleus, calculating strength distributions for several $K^{\pi}$ in ${ }^{172} \mathrm{Yb}$. We present energy-weighted sums, properties of $\gamma$-vibrational and low-energy $K^{\pi}=0^{+}$states, and the complete isovector $E 1$ strength function. The QRPA calculation reproduces the properties of the low-lying $2^{+}$ states as well or better than it typically does in spherical nuclei.
\end{abstract}

DOI: 10.1103/PhysRevC.82.034326

PACS number(s): 21.60.Jz

\section{INTRODUCTION}

The quasiparticle random-phase approximation (QRPA) $[1,2]$ has a long history in nuclear physics. Its virtues include applicability to many types of excitation across the isotopic chart, preservation of energy-weighted sum rules, and elimination of spurious motion. In addition, the QRPA has several appealing interpretations; it is both a boson approximation for collective modes and the small-amplitude limit of the time-dependent Hartree-Fock-Bogoliubov (HFB) approximation. Its downside, traditionally, has been a limited ability to describe large-amplitude motion and complicated noncollective states, deficiencies that prompted the development of several more complicated methods, as, e.g., in Refs. $[3,4]$.

Recent years, however, have seen a revival of the QRPA, despite its drawbacks. The primary reason is the increasing connection between nuclear mean-field theory and densityfunctional theory (DFT) [5,6]. The notion that Hartree-Fock or HFB calculations can be relevant beyond their naive range of validity has motivated attempts to describe a wide range of nuclear properties in mean-field theory and extensions. The QRPA is the most straightforward extension that fits into the DFT paradigm; to the extent that the energy functional used in HFB calculations is exact, the QRPA provides the exact linear (i.e., small-amplitude) response function in the adiabatic limit [6]. Combined with its other features, its connection with DFT makes the QRPA an important tool in attempts both to develop a universal nuclear energy-density functional (UNEDF) and to apply the functional to, e.g., nuclear astrophysics [7].

The prototype energy-density functional is of Skyrme form, corresponding roughly to effective interactions that have zero range, with derivatives simulating finite-range effects. In the last five or ten years, a number of groups have developed self-consistent (Q)RPA codes for use with these functionals or similar finite-range and relativistic versions, first in spherical nuclei (see Ref. [8] and references therein), and then in axially symmetric deformed nuclei [9-13]. Heavy deformed nuclei are still problematic, however. Though the deformed RPA, without pairing, is now tractable in heavy nuclei [14], a separable approximation to the Skyrme-QRPA equations has been applied in such nuclei $[15,16]$, and efficient new methods for solving the full QRPA equations are promising [14,17], the numerical complexity of deformed systems has so far limited fully self-consistent Skyrme-QRPA calculations to nuclei with $A \lesssim 40$. In this paper, we present a highly parallelized version of the Skyrme QRPA that we are beginning to apply to heavy deformed nuclei. After discussing the structure of the code and demonstrating its accuracy, we present a preliminary application to the nucleus ${ }^{172} \mathrm{Yb}$. We focus on results here, postponing most of the formalism to a later publication.

Our QRPA code comes in several versions, developed successively and checked against one another as we progressed from tests in light nuclei to full-fledged calculations in heavy nuclei. All versions treat the entire Skyrme + Coulomb functional in a way that is completely consistent with HFB calculations (restricted for the time being to "volume" pairing). In addition, all the versions preserve axial and parity symmetries and the time-reversal invariance of the ground state, and therefore require an HFB code that produces single-quasiparticle wave functions of the two variables $r \equiv$ $\sqrt{x^{2}+y^{2}}$ and $z$, with $M \equiv\left\langle j_{z}\right\rangle$ a good quantum number. Finally, all diagonalize the traditional QRPA $A, B$ matrix [1] in the " $M$ scheme," and use the rigid-rotor approximation $[18,19]$, with the deformed QRPA solutions as intrinsic states, to calculate transition strength. The versions differ, however, in the basis in which the HFB equations are solved, in the basis in which the QRPA matrix is constructed, and in the way wave functions are represented numerically. Version (a) takes quasiparticle-basis wave functions from the transformedoscillator code HFBTHO [20] (though we use the ordinary harmonic-oscillator basis), represents the wave functions on an equidistant mesh, and constructs the QRPA matrix in the quasiparticle representation. Version (b) substitutes the Vanderbilt "cylindrical-box" $B$-spline-based HFB code [21] for HFBTHO. Version (c) modifies the QRPA part of version (b) by using the canonical-quasiparticle basis, represented with $B$ splines, in place of the quasiparticle basis to speed calculation and save memory. 
The vast majority of the computing time in all versions is in the construction of the QRPA matrix, each element of which requires a series of two-dimensional integrals for the Skyrme interaction, and an additional multipole expansion for the Coulomb interaction. The set of matrix elements can be divided among many thousands of processors so that the calculation is manageable on fast supercomputers.

Section II describes tests in relatively light systems, with pairing strengths chosen so that most gaps are between 1 and $2 \mathrm{MeV}$. Section III presents an application to heavy nuclei. Section IV contains conclusions.

\section{TESTS}

To display the accuracy of our codes, we show the results of several tests in nuclei with $A<40$. We start with spurious states. A fully self-consistent and numerically perfect QRPA will completely separate spurious states from physical ones and put them at zero energy. Small numerical errors can spoil the treatment of spurious states, however; so any calculation that separates them well has passed a serious test.

Figure 1 shows transition strengths produced by the two number operators

$$
S_{k}^{\tau}=\left|\left\langle 0\left|\hat{N}_{\tau}\right| k\right\rangle\right|^{2},
$$

with $k$ labeling excitations and $\tau$ indicating protons and neutrons, to states with $K^{\pi}=0^{+}$in a calculation of ${ }^{26} \mathrm{Mg}$. To obtain these results, we use version (a) of our code; we expect it to do best in this kind of test because it allows strict control of the single-particle space, the contents of which often determine how well spurious motion is separated from physical excitations. Here we restrict ourselves to three spherical harmonic oscillator shells and use the the Skyrme interaction SkP [22]. The HFB calculation yields pairing gaps of $\Delta_{p}=1.681 \mathrm{MeV}$ (protons), $\Delta_{n}=1.426 \mathrm{MeV}$ (neutrons), and no quadrupole deformation in this small single-particle space. This result does not imply that a calculation in a larger space also gives $\beta=0$ [23]. At the QRPA level, we keep the full two-quasiparticle basis; a complete separation of the spurious $J^{\pi}=0^{+}$strength associated with the particlenumber violation requires no less. And we indeed achieve

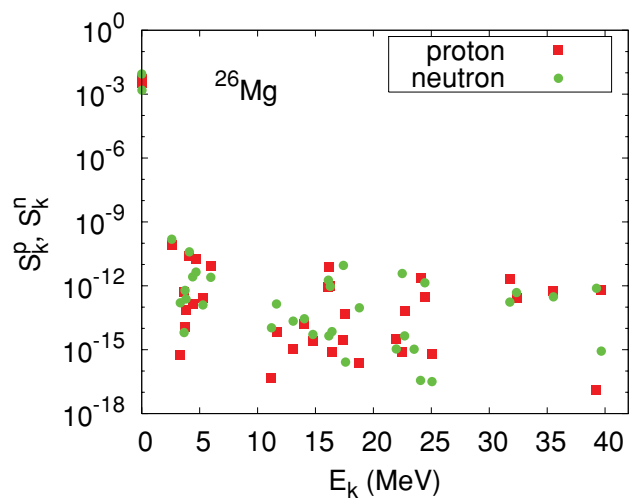

FIG. 1. (Color online) Transition strength for the proton and neutron number operators to $K^{\pi}=0^{+}$states in ${ }^{26} \mathrm{Mg}$ with the Skyrme functional SkP (see text), from version (a). essentially perfect separation. The figure shows negligible strength to all excitations except the two spurious states, which come out at $E=0.008$ and $0.024 \mathrm{MeV}$.

With versions (b) or (c), we cannot select a subspace of states that is invariant under the action of the number operator; we must truncate the space at the quasiparticle level during and after the HFB calculation rather than at the single-particle level before that calculation. When the pairing gap is nonzero, version (c), for example, employs two cutoff parameters, which we call $\left(v_{\text {cut }}^{\mathrm{pp}}\right)^{2}$ and $\left(v_{\text {cut }}^{\mathrm{ph}}\right)^{2}$. The code omits from the QRPA matrix all two-canonical-quasiparticle states (labeled by $i$ and $j$ ) with occupation probabilities $v_{i}^{2}$ and $v_{j}^{2}$ such that $v_{i}^{2}, v_{j}^{2}>1-\left(v_{\mathrm{cut}}^{\mathrm{pp}}\right)^{2}$ or $v_{i}^{2}, v_{j}^{2}<\left(v_{\mathrm{cut}}^{\mathrm{pp}}\right)^{2}$. It also omits configurations for which $v_{i}^{2} / v_{j}^{2}<\left(v_{\text {cut }}^{\mathrm{ph}}\right)^{2}$ (where $v_{i}^{2}$ is the smaller of the two). The first condition omits states that are nearly entirely two-particle or two-hole in nature; these are in a different nucleus than the ground state. The second condition omits high-energy particle-hole excitations; in those, one quasiparticle lies at much higher energy than the other and is thus nearly unoccupied. If a pairing gap is zero, an explicitly energy-based truncation scheme replaces this one.

When version (c) is applied to the excitation mode as in Fig. 1, the separation of spurious motion is very good, though not quite at the same level as that produced by version (a). We reach this conclusion by applying version (c) to the nucleus ${ }^{24} \mathrm{Mg}$, with the Skyrme interaction SLy4 [24]. The Vanderbilt HFB code, working in a cylindrical box with $r_{\max }=z_{\max }=$ $10 \mathrm{fm}$ and with a cutoff quasiparticle energy of $60 \mathrm{MeV}$, yields $\Delta_{p}=1.914 \mathrm{MeV}, \Delta_{n}=2.164 \mathrm{MeV}$, and $\beta=0.433$. In the QRPA calculation, to obtain a Hamiltonian matrix of easily manageable size, we use truncation parameters $\left(v_{\text {cut }}^{\mathrm{pp}}\right)^{2}=10^{-6}$ and $\left(v_{\mathrm{cut}}^{\mathrm{ph}}\right)^{2}=10^{-9}$. The resulting spurious state energies are 0.654 and $2.012 \mathrm{MeV}$, and the maximum $S_{k}^{\tau}$ of real states is less than $0.5 \%$ of the spurious strength. Changing the truncation parameter $\left(v_{\text {cut }}^{\mathrm{pp}}\right)^{2}$ to $10^{-5}$ makes little difference.

Next, we turn to spurious rotation, arising in the $K^{\pi}=1^{+}$ channel. Here the nucleus must be deformed. With version (a) and $\mathrm{SkP}$ as before but in ${ }^{24} \mathrm{Mg}$, our HFB solution with four spherical oscillator shells, yields $\beta=0.28, \Delta_{p}=0.034 \mathrm{MeV}$, and $\Delta_{n}=0.131 \mathrm{MeV}$. Figure 2 displays the resulting transition strengths for the operator $J_{-1} \equiv J_{x}-i J_{y}$. Most of the

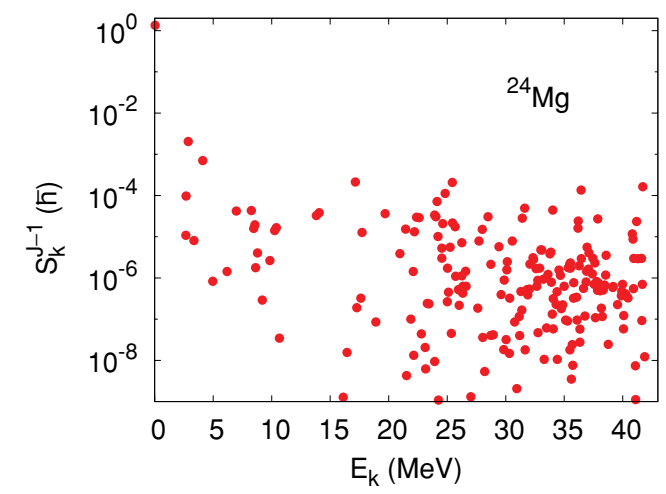

FIG. 2. (Color online) Transition strength for the operator $J_{-1}$ to $K^{\pi}=1^{+}$states in ${ }^{24} \mathrm{Mg}$ with $\mathrm{SkP}$ obtained by version (a). 
strengths are four orders of magnitude smaller than that of the spurious state at $E=0.045 \mathrm{MeV}$.

For this one mode, version (c) does not give good results. We use the same box-HFB solution of ${ }^{24} \mathrm{Mg}$ as for $K^{\pi}=$ $0^{+}$just discussed, and choose truncation parameters $\left(v_{\text {cut }}^{\mathrm{pp}}\right)^{2}=$ $10^{-4}$ and $\left(v_{\text {cut }}^{\mathrm{ph}}\right)^{2}=10^{-13}$. The resulting spurious state energy is $1.731 \mathrm{MeV}$, a rather large value, and although nearly all physical states have negligible strength, a few around $E=10$ and $20 \mathrm{MeV}$ have significant strength. The poor performance is due to the rotationally asymmetric quasiparticle space that the cylindrical-box boundary conditions create; changing the truncation parameters has little effect. The space of spherical oscillator states we use with version (a) is invariant under rotation and therefore far better able to separate collective rotation.

Finally, we examine spurious translational motion. Here, an oscillator basis for HFB is far from optimal. With version (a) in ${ }^{16} \mathrm{O}$, the spurious state energy was nearly $2.5 \mathrm{MeV}$ even when 40 oscillator shells were included. We therefore choose here to test versions (b) and (c), which turn out to give identical strength functions. We choose the nucleus ${ }^{26} \mathrm{Mg}$, the same box as in the tests already discussed, the Skyrme functional SLy4, and a pairing strength $V=-140.348 \mathrm{MeV} \mathrm{fm}^{3}$, with the quasiparticle cutoff in the HFB code increased to $300 \mathrm{MeV}$. These parameters yield $\beta=-0.27, \Delta_{p}=1.365 \mathrm{MeV}$, and $\Delta_{n}=0.002 \mathrm{MeV}$. Figure 3 shows isoscalar (IS) $E 1$ transition strengths, for which the excitation operator is $\sum_{i=1}^{A} r_{i}^{3} Y_{1 \mu}\left(\Omega_{i}\right)$, to states with $K^{\pi}=0^{-}$. The figure contains two sets of lines, the second of which adds a correction term to the IS operator (via the prescription of Ref. [12]) to remove residual spurious strength from physical excitations. The difference between the two sets is very small in all the physical states shown, and completely negligible in higher-energy states. In addition, the smoothed version of the spectrum proves to be stable against changes in the truncation parameters. This test and those presented earlier show that we can handle spurious motion in this mass region without trouble.

Since we have done extensive calculations with a spherical $J$-scheme code over the past few years [25], we can test our current codes further by comparing their results with those

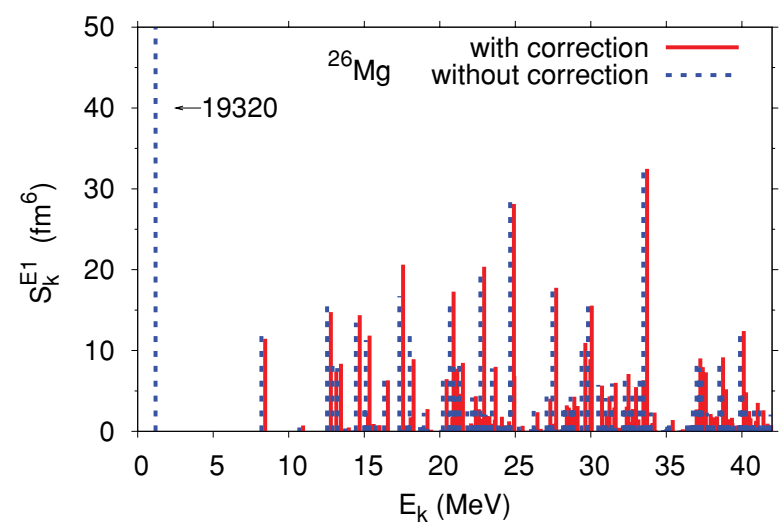

FIG. 3. (Color online) IS $E 1$ transition strength to $K^{\pi}=0^{-}$states in ${ }^{26} \mathrm{Mg}$ with SLy4, from version (b). Solid lines represent strength that is corrected to eliminate residual spurious contributions.
TABLE I. Comparison of energies and (corrected) IS E 1 strengths for the three lowest-lying $J^{\pi}=1^{-}$states in ${ }^{16} \mathrm{O}$, calculated with spherical ( $J$-scheme) code and the current $(M$-scheme) code and SLy4. The correction barely changes the strength for the two physical excited states, but reduces that of the spurious (lowest) state by many orders of magnitude.

\begin{tabular}{ccccc}
\hline \hline \multicolumn{2}{c}{$J$ scheme, $J^{\pi}=1^{-}$} & & \multicolumn{2}{c}{$M$ scheme, $K^{\pi}=0^{-}$} \\
\cline { 5 - 6 } \begin{tabular}{c}
$E$ \\
\cline { 5 - 6 }$(\mathrm{MeV})$
\end{tabular} & $\begin{array}{c}S_{\text {cor }}^{\mathrm{ISE1}} \\
\left(\mathrm{fm}^{6}\right)\end{array}$ & & $\begin{array}{c}E \\
(\mathrm{MeV})\end{array}$ & $\begin{array}{c}S_{\text {cor }}^{\mathrm{ISE} 1} \\
\left(\mathrm{fm}^{6}\right)\end{array}$ \\
\hline 0.323 & $7.051 \times 10^{-5}$ & & 0.472 & $1.298 \times 10^{-4}$ \\
7.500 & $1.461 \times 10$ & & 7.440 & $1.433 \times 10$ \\
10.610 & $5.739 \times 10^{-2}$ & & 10.681 & $4.283 \times 10^{-2}$ \\
\hline \hline
\end{tabular}

obtained in the $J$ scheme. Table I shows energies and IS $E 1$ transition strengths (with the correction mentioned above) for the three lowest $J^{\pi}=1^{-}$levels, along with the corresponding $K^{\pi}=0^{-}$energies and strengths from version (b) of our current code, in the spherical nucleus ${ }^{16} \mathrm{O}$. The two codes take wave functions from entirely different HFB codes: a slightly modified version of HFBRAD [22] called HFBMARIO for the spherical QRPA and the Vanderbilt HFB code for the deformed QRPA. The first state on each side of the table is the spurious state, with very small strength because of the correction. The next two, both genuine excitations, are nearly the same in both energy and strength in the two calculations. The full strength function, folded with a Lorentzian of width $3 \mathrm{MeV}$ [see in Eq. (1) in Ref. [8]] displayed in Fig. 4, shows the same level of agreement. The very small differences in the continuum are due to differing box boundary conditions: the spherical calculation is in a spherical box with radius $20 \mathrm{fm}$ and the deformed calculation is in the same cylindrical box we used for ${ }^{26} \mathrm{Mg}$.

Before moving to heavy nuclei, we display the results of one more test-this time simultaneously checking the Vanderbilt HFB code [21] underlying our deformed QRPA, and our procedure for constructing the canonical basis for version (c) by diagonalizing the density matrix (which is the same as in Ref. [8]). Figure 5 displays the proton $0 p_{3 / 2}\left(j_{z}=3 / 2\right)$ canonical basis wave function in ${ }^{22} \mathrm{O}$ produced by both the spherical and deformed procedures. The agreement is perfect

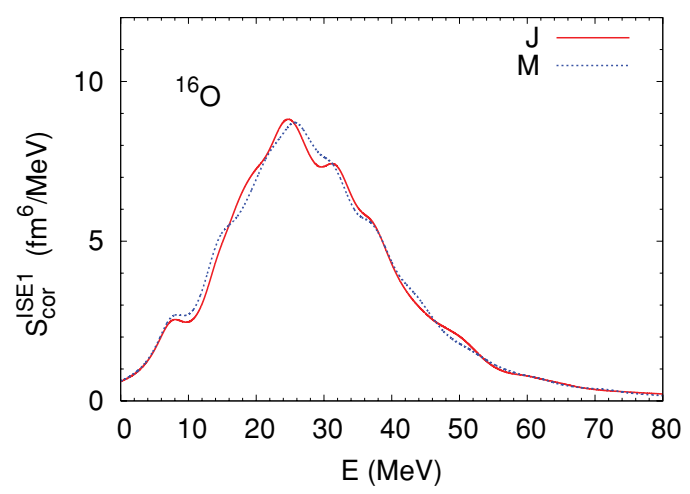

FIG. 4. (Color online) Full IS $E 1$ strength function corresponding to Table I. The letters $J$ and $M$ denote the $J$ - and $M$-scheme calculations. 


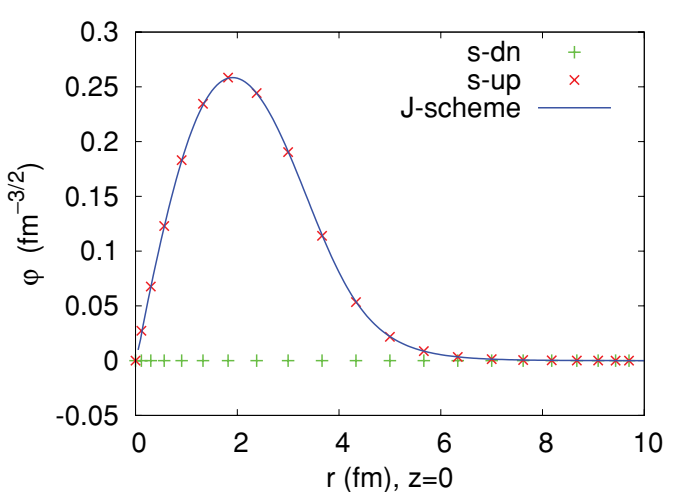

FIG. 5. (Color online) Comparison of proton $0 p_{3 / 2}\left(j_{z}=3 / 2\right)$ canonical-basis wave function produced by $J$ - and $M$-scheme codes for ${ }^{22} \mathrm{O}$ at $z=0$. The labels s-dn and s-up denote the spin-down and spin-up components in the $M$-scheme calculation. In the $J$ scheme, the spin-down component is identically zero. See, e.g., Ref. [22] for details on the spin-specific components of $J$-scheme wave functions.

and far from trivial. Though $\Delta_{p}=0$ in this calculation, we still construct the density matrix from the Vanderbilt-HFB output and diagonalize it to obtain the canonical wave function. (There is no arbitrariness in this wave function because only one proton $p_{3 / 2}$ state is occupied in oxygen.) The neutron bound-state wave functions produced by the two procedures, though we do not display them, agree equally well.

\section{HEAVY NUCLEI}

Having thoroughly tested several versions of the deformed QRPA, we apply version (c) to the nucleus ${ }^{172} \mathrm{Yb}$. The use of the canonical basis makes version (c) faster than version (b), and the use of box boundary conditions makes it better able to represent the continuum than version (a). Though we may pay a price in the $K^{\pi}=1^{+}$channel, we should be better off everywhere else.

We set up the HFB calculation as follows: we use a "box" with $r_{\max }=z_{\max }=20 \mathrm{fm}$, cut off the quasiparticle spectrum at $60 \mathrm{MeV}$, and take the maximum $z$ component of the quasiparticle angular momentum to be $19 / 2$; these parameters define a single-quasiparticle space with 4648 proton states and 5348 neutron states. We use the Skyrme functional SkM* [26] with volume pairing strengths $V_{p}=-218.521 \mathrm{MeV} \mathrm{\textrm {fm } ^ { 3 }}$ and $V_{n}=-176.364 \mathrm{MeV} \mathrm{fm}{ }^{3}$ (determined from measured odd-even mass differences). The calculation yields $\Delta_{p}=$ $1.248 \mathrm{MeV}, \Delta_{n}=0.773 \mathrm{MeV}$, and $\beta=0.34$.

In the QRPA, we truncate two-canonical-quasiparticle configurations by choosing $\left(v_{\text {cut }}^{\mathrm{pp}}\right)^{2}=10^{-6}$ and $\left(v_{\text {cut }}^{\mathrm{ph}}\right)^{2}=10^{-10}$. The values of these parameters are designed to yield a manageable calculation (a QRPA matrix whose size, while depending on multipolarity, is typically about 160000 by 160000 ) while inducing only small errors. (The accuracy with which we treat canonical wave functions results in a very close correlation between the cutoff parameters and the dimension of the resulting QRPA matrix. When changing $\left(v_{\mathrm{cut}}^{\mathrm{ph}}\right)^{2}$ from 0 to $10^{-15}$ and then $10^{-13}$, the dimension decreases from 356126

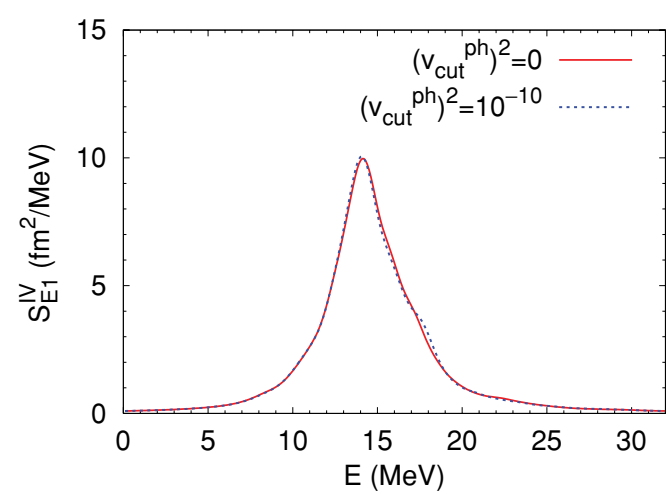

FIG. 6. (Color online) Comparison of isovector (IV) $E 1$ strength functions in a fictitious spherical ${ }^{172} \mathrm{Yb}$ nucleus, with particle-hole cutoffs $\left(v_{\text {cut }}^{\text {ph }}\right)^{2}$ of $10^{-10}$ and zero. We assume a spherical nucleus in order to set $\left(v_{\text {cut }}^{\mathrm{ph}}\right)^{2}$ to zero while keeping the calculation manageable. We use the $J$-scheme code [8] with a box radius of $20 \mathrm{fm}$, the Skyrme functional $\mathrm{SkM}^{*}$, and $\left(v_{\mathrm{cut}}^{\mathrm{pp}}\right)^{2}$ set to $\left(v_{\mathrm{cut}}^{\mathrm{ph}}\right)^{2}$. The self-consistent pairing gaps are between 1.3 and $1.4 \mathrm{MeV}$ for both protons and neutrons, and we use a folding width of $1 \mathrm{MeV}$ to produce the figure. Changing the box size to 15 or $17.5 \mathrm{fm}$ makes almost no difference in the strength function.

to 303996 and then to 233314 for $K^{\pi}=1^{-}$.) Our experience with accurate calculations in spherical nuclei $[8,25]$ and in a fictitious spherical version of ${ }^{172} \mathrm{Yb}$ with no occupation cutoff, see Fig. 6, suggests that the calculations here will be accurate as well; the only way in which they are slightly inferior is through the parameter $\left(v_{\text {cut }}^{\mathrm{pp}}\right)^{2}$, which in spherical nuclei we took to be $10^{-12}$ for channels with spurious states and $10^{-8}$ otherwise. Calculations of similar size in deformed nuclei with $\left(v_{\text {cut }}^{\mathrm{pp}}\right)^{2}=10^{-4}$ and $\left(v_{\text {cut }}^{\mathrm{ph}}\right)^{2}=10^{-13}$ in the $K^{\pi}=0^{+}$and $1^{-}$ channels give nearly the same results as those reported here, indicating that neither truncation causes significant errors.

Table II shows energy-weighted sums, alongside values obtained from sum rules, for IS and isovector (IV) electric

TABLE II. Energy-weighted sums for strength functions, in our QRPA calculations and from analytical sum-rule expressions. We include QRPA states with up to $90 \mathrm{MeV}$ of excitation energy. The units of the IS $E 1$ sum are $\mathrm{MeV} \mathrm{fm}^{6}$, and those of the IV $E 1$ sum are $\mathrm{MeV} \mathrm{fm}{ }^{2}$. Those of all $E 2$ sums are $\mathrm{MeV} \mathrm{fm}^{4}$. The IS $E 1$ strength has been corrected to remove spurious components. Contribution of $-K$ is not included in $K \neq 0$.

\begin{tabular}{cccc}
\hline \hline $\begin{array}{c}\text { Transition } \\
\text { operator }\end{array}$ & $\begin{array}{c}K^{\pi} \\
\text { of solution }\end{array}$ & QRPA & Analytical \\
\hline IS $E$ 1 & $1^{-}$ & 1043560 & 1042413 \\
IV $E 1$ & $1^{-}$ & 289.819 & 285.764 \\
IS $E 1$ & $0^{-}$ & 2015266 & 2019465 \\
IV $E 1$ & $0^{-}$ & 291.859 & 285.764 \\
IS $E 2$ & $2^{+}$ & 64700 & 63877 \\
IV E2 & $2^{+}$ & 20284 & 20076 \\
IS $E 2$ & $1^{+}$ & 76159 & 88197 \\
IV E2 & $1^{+}$ & 28517 & 28174 \\
IS $E 2$ & $0^{+}$ & 97886 & 96867 \\
IV E2 & $0^{+}$ & 31271 & 30874 \\
\hline \hline
\end{tabular}


TABLE III. Energies and $B\left(E 2 ; 0^{+} \rightarrow 2^{+}\right)$'s for the $\gamma$-vibrational and " $\beta$-vib." states of ${ }^{172} \mathrm{Yb}$. Experimental data are from Ref. [27] (see also Ref. [28]). For the definition of $B(E 2)$, see, e.g., Ref. [29].

\begin{tabular}{cccc}
\hline \hline & & Expt. & Calc. \\
\hline$\gamma$-vib. & $E(\mathrm{MeV})$ & 1.466 & 2.261 \\
& $B(E 2)\left(e^{2} \mathrm{~b}^{2}\right)$ & $0.043+5-15$ & 0.041 \\
" $\beta$-vib." & $E(\mathrm{MeV})$ & 1.117 & 1.390 \\
& $B(E 2)\left(e^{2} \mathrm{~b}^{2}\right)$ & 0.008117 & 0.00495 \\
\hline \hline
\end{tabular}

operators in all ${ }^{172} \mathrm{Yb}$ channels that we calculate. The differences between the QRPA sums and the sum rules are less than about $2 \%$ except in the IS $K^{\pi}=1^{+}$channel, where as seen earlier, we are unable to adequately separate the spurious rotational state in our deformed box. In other channels, the spurious mode is under better control. In the IS $0^{-}$and $1^{-}$channels, we have some contamination at low energies, but it is weak enough that the subtraction procedure of Ref. [12] restores the sum rule nearly exactly. In the $0^{+}$channel, as the table shows, the separation is quite good even without subtraction. The $1^{+}$channel can be corrected as well, but doing so requires a numerical procedure that we have not yet implemented.

The energies of the spurious states, for completeness, are 0.318 and $1.078 \mathrm{MeV}$ for $K^{\pi}=0^{+}, 0.963 \mathrm{MeV}$ for $K^{\pi}=1^{+}$, $1.480 \mathrm{MeV}$ for $K^{\pi}=0^{-}$, and $1.506 \mathrm{MeV}$ for $K^{\pi}=1^{-}$.

The accuracy of the energy-weighted sums in the $K^{\pi}=0^{+}$ and $2^{+}$channels indicates that our approach is reliable for lowlying quadrupole shape vibrations. Table III shows the energies and $B\left(E 2 ; 0^{+} \rightarrow 2^{+}\right)$'s of both the $\gamma$-vibrational $K^{\pi}=2^{+}$ state and a low-energy $K^{\pi}=0^{+}$state with a significant $B(E 2)$ that we denote by " $\beta$-vib." The quotation marks indicate that, unlike the clear-cut $\gamma$-vibrational state, the $0^{+}$state has a somewhat smaller $B(E 2)$ than is typical of vibrational modes. Both states have been studied experimentally, e.g., in Ref. [27]. The particle-number strength of the " $\beta$-vib." state is $0.04 \%$ of that of the spurious state, indicating that we need not worry about contamination.

The agreement of both the energies of these low-lying states and their transition strengths with measured values is at a level that is typical of QRPA calculations in spherical nuclei. In Ref. [30], we investigated a large set of such nuclei, characterizing the quality of the QRPA by two quantities:

$$
\begin{aligned}
R_{E} & \equiv \ln \left(E_{\text {calc }} / E_{\text {expt }}\right), \\
R_{Q} & \equiv \ln \sqrt{B(E 2)_{\text {calc }} / B(E 2)_{\text {expt }}},
\end{aligned}
$$

where suffixes calc and expt denote calculated and experimental. The results in Table III correspond to $R_{E}=0.43$ and $R_{Q}=-0.03$ for the $\gamma$ vibration, and $R_{E}=0.22$ and $R_{Q}=-0.25$ for " $\beta$-vib." The histograms in Figs. 4 and 9 of Ref. [30] show these values to be near the most common values in spherical nuclei.

Figure 7 displays the IV $E 1$ strength function. The thick curve is the sum of strengths in all channels and can be compared with experimental data. The peaks of the $K^{\pi}=0^{-}$ and $1^{-}$distributions in Fig. 7 lie at different energies, as is

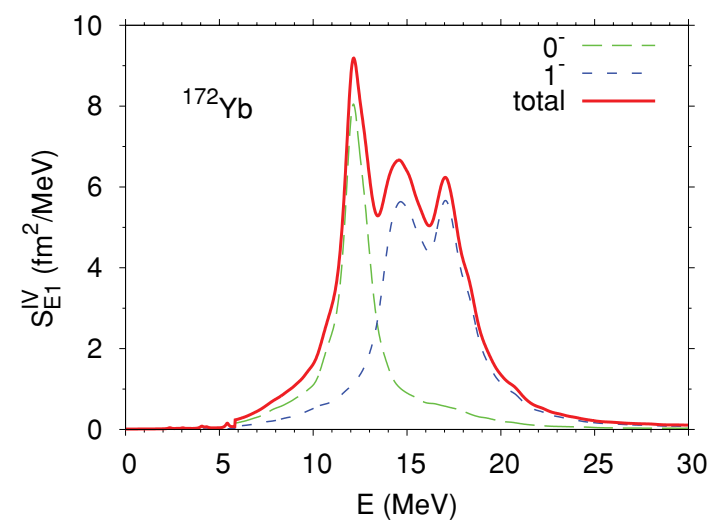

FIG. 7. (Color online) Predicted IV E1 strength function in ${ }^{172} \mathrm{Yb}$, with a folding width of $0.5 \mathrm{MeV}$ ( $0.1 \mathrm{MeV}$ for discrete states). $0^{-}$and $1^{-}$indicate $K^{\pi}$ components (the curve for $K^{\pi}=1^{-}$includes contributions from $K^{\pi}=-1^{-}$).

often the case in deformed nuclei. Though an experimental group reports a pygmy resonance at 3-4 $\mathrm{MeV}$ [31], we see no indication of one in our calculation. It has been suggested that in spherical $\mathrm{Sn}$ isotopes such resonances involve configurations beyond the natural ambit of the QRPA [3,4], but the issue is unresolved; and in deformed nuclei, it has not been systematically investigated.

\section{CONCLUSIONS}

In summary, we have developed three related SkyrmeHFB + QRPA codes, aimed at calculations in axially symmetric even-even nuclei throughout the isotopic chart. We rigorously tested the accuracy of the codes in $\mathrm{Mg}$ isotopes, paying particular attention to spurious states. In heavier nuclei, version (c) appears to perform as well as in light systems, provided we work in larger but still manageable spaces. The agreement of energy-weighted sums with sum rules indicates that (1) in channels with no spurious modes (e.g., $K^{\pi}=2^{+}$), our code is quite accurate, (2) in the $K^{\pi}=0^{+}$channel, spurious admixtures are negligible, and (3) in the IS $K^{\pi}=0^{-}$, and $1^{-}$channels [and perhaps the $1^{+}$channel as well with version (a)], admixtures can be effectively removed. Our immediate plans are to systematically investigate the ability of modern density functionals, together with the QRPA, to describe $\beta$ and $\gamma$ vibrations in rare-earth nuclei.

\section{ACKNOWLEDGMENTS}

This work was supported by the UNEDF SciDAC Collaboration under DOE Grant No. DE-FC02-07ER41457 and by the National Science Foundation through Teragrid resources provided by the National Institute for Computational Sciences. We are indebted to Profs. V. E. Oberacker and A. S. Umar for giving us their HFB code and for technical support. We used computers at the National Energy Research Scientific Computing Center, the National Center for Computational Sciences, and the University of North Carolina at Chapel Hill. 
[1] P. Ring and P. Schuck, The Nuclear Many-Body Problem (Springer-Verlag, New York, 1980).

[2] J.-P. Blaizot and G. Ripka, Quantum Theory of Finite Systems (MIT, Cambridge, MA, 1986).

[3] N. Tsoneva, H. Lenske, and Ch. Stoyanov, Phys. Lett. B 586, 213 (2004).

[4] D. Sarchi, P.-F. Bortignon, and G. Colò, Phys. Lett. B 601, 27 (2004).

[5] I. Z. Petkov and M. V. Stoitsov, Nuclear Density Functional Theory (Clarendon, Oxford, 1991).

[6] A Primer in Density Functional Theory, edited by C. Fiolhais, F. Nogueira, and M. Marques (Springer, Berlin, 2003).

[7] M. Arnould and S. Goriely, Phys. Rep. 384, 1 (2003).

[8] J. Terasaki, J. Engel, M. Bender, J. Dobaczewski, W. Nazarewicz, and M. Stoitsov, Phys. Rev. C 71, 034310 (2005).

[9] M. Yamagami and N. Van Giai, Phys. Rev. C 69, 034301 (2004).

[10] D. Pena Arteage and P. Ring, Phys. Rev. C 77, 034317 (2008).

[11] S. Péru and H. Goutte, Phys. Rev. C 77, 044313 (2008).

[12] K. Yoshida and N. V. Giai, Phys. Rev. C 78, 064316 (2008).

[13] C. Losa, A. Pastore, T. Døssing, E. Vigezzi, and R. A. Broglia, Phys. Rev. C 81, 064307 (2010).

[14] T. Inakura, T. Nakatsukasa, and K. Yabana, Phys. Rev. C 80, 044301 (2009).

[15] V. O. Nesterenko, W. Kleinig, J. Kvasil, P. Vesely, P.-G. Reinhard, and D. S. Dolci, Phys. Rev. C 74, 064306 (2006).
[16] A. P. Severyukhin, V. V. Voronov, and N. V. Giai, Phys. Rev. C 77, 024322 (2008).

[17] J. Toivanen, B. G. Carlsson, J. Dobaczewski, K. Mizuyama, R. R. Rodríguez-Guzmán, P. Toivanen, and P. Veselý, Phys. Rev. C 81, 034312 (2010).

[18] A. Bohr and B. R. Mottelson, Nuclear Structure (Benjamin, Reading, 1975), Vol. II.

[19] V. G. Soloviev and N. Yu. Shirikova, Z. Phys. A 334, 149 (1989).

[20] M. V. Stoitsov et al., Comput. Phys. Commun. 167, 43 (2005).

[21] A. Blazkiewicz, V. E. Oberacker, A. S. Umar, and M. Stoitsov, Phys. Rev. C 71, 054321 (2005).

[22] J. Dobaczewski, H. Flocard, and J. Treiner, Nucl. Phys. A 422, 103 (1984).

[23] M. V. Stoitsov, J. Dobaczewski, W. Nazarewicz, S. Pittel, and D. J. Dean, Phys. Rev. C 68, 054312 (2003).

[24] E. Chabanat et al., Nucl. Phys. A 635, 231 (1998).

[25] J. Terasaki and J. Engel, Phys. Rev. C 74, 044301 (2006).

[26] J. Bartel et al., Nucl. Phys. A 386, 79 (1982).

[27] C. Fahlander et al., Nucl. Phys. A 541, 157 (1992).

[28] [http://www.nndc.bnl.gov].

[29] A. Bohr and B. R. Mottelson, Nuclear Structure (Benjamin, New York, 1969), Vol. I.

[30] J. Terasaki, J. Engel, and G. F. Bertsch, Phys. Rev. C 78, 044311 (2008).

[31] A. Voinov, M. Guttormsen, E. Melby, J. Rekstad, A. Schiller, and S. Siem, Phys. Rev. C 63, 044313 (2001). 